\title{
Phase Equilibria and Crystal Growth in the Alkali Antimonate Systems $\mathrm{Sb}_{2} \mathrm{O}_{4}-\mathrm{NaSbO}_{3}, \mathrm{Sb}_{2} \mathrm{O}_{4}-\mathrm{KSbO}_{3}$, and $\mathrm{Sb}_{2} \mathrm{O}_{4}-\mathrm{NaSbO}_{3}-\mathrm{NaF}^{*}$
}

\author{
J. L. Waring, R. S. Roth, H. S. Parker, and W. S. Brower, Jr. \\ Institute for Materials Research, National Bureau of Standards, Washington, D.C. 20234 \\ (July 8, 1976)
}

\begin{abstract}
Phase equilibrium diagrams have been constructed from experimental data for the systems $\mathrm{Sb}_{2} \mathrm{O}_{4}-\mathrm{NaSb0}_{3}, \mathrm{Sb}_{2} 0_{4}-\mathrm{KSb0}_{3}$, and $\mathrm{Sb}_{2} \mathrm{O}_{4}-\mathrm{NaSb0}_{3}-\mathrm{NaF}$. The system $\mathrm{Sb}_{2} \mathrm{O}_{4}-\mathrm{NaSbO}_{3}$ contains only an intermediate pyrochlore type solid solution with a maximum melting point of $14900^{\circ} \mathrm{C}$ at a $\mathrm{Na}: \mathrm{Sb}$ atom ratio of $3: 5$. The $\mathrm{Sb}_{2} \mathrm{O}_{4}-\mathrm{KSbO}_{3}$ system contains in addition to the pyrochlore phase a compound $3 \mathrm{~K}_{2} \mathrm{O} \cdot 5 \mathrm{Sb}_{2} \mathrm{O}_{5}$ which melts congruently at about $1450{ }^{\circ} \mathrm{C}$ and two polymorphs of $\mathrm{K}_{2} \mathrm{O} \cdot 2 \mathrm{Sb}_{2} \mathrm{O}_{5}$. The low temperature form of $\mathrm{K}_{2} \mathrm{O} \cdot 2 \mathrm{Sb}_{2} \mathrm{O}_{5}$ was found to be monoclinic P2 $1 / \mathrm{c}$ with $a=7.178, b=13.378, c=11.985 \mathrm{~A}, \beta=124^{\circ} 10^{\prime}$. The melting point of $\mathrm{Sb}_{2} \mathrm{O}_{4}$ was found to be $1350 \pm 5{ }^{\circ} \mathrm{C}$ and $\mathrm{NaSbO}_{3}$ and $\mathrm{KSbO}_{3}$ both melt congruently at $1555 \pm 5{ }^{\circ} \mathrm{C}$ and $1410 \pm 5{ }^{\circ} \mathrm{C}$ respectively. The previously reported cubic form of $\mathrm{KSbO}_{3}$ was found to be a $\mathrm{K}^{+}$deficient phase stabilized by reaction with atmospheric moisture. A similar cubic phase which appears to be a good $\mathrm{Na}^{+}$ion conductor can be synthesized in the ternary system $\mathrm{NaSbO}_{3}-\mathrm{Sb}_{2} \mathrm{O}_{4}-\mathrm{NaF}$.
\end{abstract}

Key words: Alkali antimonates; crystal growth; ionic conductors; potassium antimonate; sodium antimonate; sodium antimony oxyfluoride.

\section{Introduction}

The search for potential candidates for ionic conductors together with the lack of detailed phase equilibrium data has served as an impetus to investigate the alkali antimonate systems.

In the system $\mathrm{Na}_{2} \mathrm{O}-\mathrm{Sb}_{2} \mathrm{O}_{4}-\mathrm{O}_{2}$ the compound $\mathrm{NaSbO}_{3}$ was reported to occur by Schrewelius [1] ${ }^{1}$ and to be hexagonal with an ilmenite structure, $a=5.316$, $c=15.95 \AA$ A. A pyrochlore solid solution was found to occur by Steward and Knop [2]. No melting data was previously reported.

In the system $\mathrm{K}_{2} \mathrm{O}-\mathrm{Sb}_{2} \mathrm{O}_{4}-\mathrm{O}_{2}$ the compound $\mathrm{KSbO}_{3}$ with an ilmenite structure has been previously reported by Spiegelberg [3]. A body centered cubic solid solution phase originally reported as $\mathrm{KSbO}_{3}$ [3] has been found to occur metastably in the system. The $3 \mathrm{~K}_{2} \mathrm{O}: 5 \mathrm{Sb}_{2} \mathrm{O}_{5}$ compound was originally reported by Aurivillius [4] and this phase, orthorhombic with $a=24.274, b=7.157, c=7.334 \mathrm{~A}$, space group $\mathrm{Pbam}$ and the new compound $\mathrm{K}_{2} \mathrm{O}: 2 \mathrm{Sb}_{2} \mathrm{O}_{5}$, monoclinic with $a=19.473, b=7.452, c=7.198 \mathrm{~A}, \beta=94^{\circ} 54.4^{\prime}$ space group $\mathrm{C} 2 / \mathrm{m}$ were reported by $\mathrm{H} . \mathrm{Y}-\mathrm{P}$ Hong [5].

Two stable polymorphs of $\mathrm{Sb}_{2} \mathrm{O}_{4}$ have been reported in the literature. They are $\alpha-\mathrm{Sb}_{2} \mathrm{O}_{4}$ which is orthorhombic [6] $a=5.436, b=11.76$ and $c=4.81 \mathrm{~A}$

${ }^{*}$ This work was supported in part by NASA Contract No. C-50821-C and a shorter version of this paper has been included as part of the NASA Report NBSIR $75-754$.

1 Figures in brackets indicate the literature references at the end of this paper. and $\beta-\mathrm{Sb}_{2} \mathrm{O}_{4}$ which is monoclinic [7] $a=11.905$, $b=4.834, c=5.383 \mathrm{~A}, \beta=101^{\circ} 22^{\prime}$. In the following discussion all ratios $(1: 3,3: 5$, etc.) refer to the alkali/metal atom ratio rather than to the particular starting material that may have been used.

\section{Specimen Preparation and Test Methods}

In order to minimize the effect of foreign anion contamination in $\mathrm{Sb}_{2} \mathrm{O}_{4}$, high purity antimony metal was ground and oxidized on Pt setters in air at elevated temperatures. It was found that the formation of a thin antimony oxide coating at $450^{\circ} \mathrm{C}$ prevented further surface reaction of antimony with the platinum. Once this coating was formed the temperature could be raised to $500{ }^{\circ} \mathrm{C}$ for relatively rapid oxidation without reaction with the platinum setter. Spectrographic analysis of $\mathrm{Sb}_{2} \mathrm{O}_{4}$ indicated that platinum contamination was only $2 \mathrm{ppm}$. The only other metals found in quantities greater than the detectable limit were traces of $\mathrm{Zr}$ and $\mathrm{Tb}$.

Mixtures of $\mathrm{Sb}_{2} \mathrm{O}_{4}$ with sodium or potassium carbonate were prepared by weighing to the nearest $\pm 0.1 \mathrm{mg}$ in sufficient quantities to yield a $1 \mathrm{~g}$ batch. No corrections were made for percentage purity except loss on ignition. Each batch was hand mixed under acetone with a mortar and pestle. The mixtures were placed on setters fabricated from platinum foil and calcined in air at $500^{\circ} \mathrm{C}$ for $60 \mathrm{hs}$. Following this preliminary calcine the mixtures 
received a second calcine at $700{ }^{\circ} \mathrm{C}$ for $60 \mathrm{hs}$. In the $\mathrm{K}_{2} \mathrm{O}-\mathrm{Sb}_{2} \mathrm{O}_{4}$ system the specimens received a third calcining at higher temperatures in a small platinum tube for $1 \mathrm{~h}$. To minimize volatility at higher temperatures, sealed platinum tubes approximately $2 \mathrm{~cm}$ long were employed for all succeeding experiments unless otherwise stated. About one-third of the volume was occupied by the specimen and the remainder of the tube was flattened prior to sealing. At elevated temperatures the expansion of the flattened portion of the tube provided the necessary additional volume for expansion of the vapors without rupture. The actual pressure inside the tube is unknown. At elevated temperatures the time of the experiment was shortened to minimize "soaking in" of $\mathrm{Sb}_{2} \mathrm{O}_{4}$ into the platinum. By employing this procedure it was felt that the best approach to equilibrium was achieved. Sub-solidus and melting point values were obtained by quenching specimens sealed in platinum tubes and examining them at room temperature.

An electrically heated vertical tube furnace was used for quenching. The temperature was controlled to about $\pm 2{ }^{\circ} \mathrm{C}$. Temperatures were measured with a $\mathrm{Pt}$ versus $\mathrm{Pt} 10$ percent $\mathrm{Rh}$ thermocouple which was calibrated several times during the course of the work. Due to the marked volatility of the antimonates and the reactivity of the alkaline materials at elevated temperatures, thermocouple contamination sometimes resulted. To minimize this contamination problem the thermocouples were changed frequently. The overall accuracy of the reported temperature is estimated to be about $\pm 5^{\circ} \mathrm{C}$.

The first sign of glazing of the specimen surface established the solidus values. The few liquidus values that are reported were established by the formation of a concave meniscus. No attempt was made to obtain liquidus values in the $\mathrm{Sb}_{2} \mathrm{O}_{4}$-rich portion of these systems because of the high vapor pressure.

Equilibrium is generally considered to have been obtained when x-ray diffraction patterns of specimens successively heated for longer times and/or at higher temperatures show no change. X-ray powder diffraction patterns were made using a high angle recording Geiger counter diffractometer and nickelfiltered copper radiation with a scan rate of $1 / 4^{\circ}$ $2 \theta / \mathrm{min}$ and a chart speed of $1 / 4 \mathrm{in} / \mathrm{min}$. The unit cell dimensions reported can be considered accurate to about \pm 5 in the last decimal place listed.

\section{The System $\mathrm{Sb}_{2} \mathrm{O}_{4}-\mathrm{NaSbO}_{3}$}

The system between the compositional limits of $\mathrm{NaSbO}_{3}$ and $\mathrm{Sb}_{2} \mathrm{O}_{4}$ has been examined in detail. The phase equilibrium diagram, figure 1, has been constructed from the data given in table 1 . When $\mathrm{Sb}_{2} \mathrm{O}_{4}$ is reacted at low temperature $\left(500-1000^{\circ} \mathrm{C}\right)$ with alkali carbonate it generally loses $\mathrm{CO}_{2}$ and gains oxygen from the atmosphere to satisfy the equilibrium conditions of the phases formed, which may involve oxidation of the antimony ions. It is therefore understood that the phase diagrams determined in the antimonate systems reported here may not be strictly binary.

The compound $\mathrm{NaSbO}_{3}$ (ilmenite-type) was found in this work to melt at about $1555 \pm 5{ }^{\circ} \mathrm{C}$. An intermediate pyrochlore solid solution exists from about $37.5 \mathrm{~mol}$ percent $\mathrm{Na}_{2} \mathrm{O}: 62.5 \mathrm{~mol}$ percent $\mathrm{Sb}_{2} \mathrm{O}_{4}$ to 24 mol percent $\mathrm{Na}_{2} \mathrm{O}: 76$ mol percent $\mathrm{Sb}_{2} \mathrm{O}_{4}$ at $1350{ }^{\circ} \mathrm{C}$. The $1: 3$ composition probably does not really correspond structurally to $\left[\mathrm{NaSb}^{+3}\right] \mathrm{Sb}_{2}{ }^{+5} \mathrm{O}_{7}$ although the $3: 5$ composition may be written as $\left[\mathrm{Na}_{1.5} \mathrm{Sb}_{0.5}^{+3}\right] \mathrm{Sb}_{2}{ }^{+5} \mathrm{O}_{6.5}$ - see section 6.1. The $3 \mathrm{Na}_{2} \mathrm{O}$ : $5 \mathrm{Sb}_{2} \mathrm{O}_{4}$ composition apparently melts congruently at $1490 \pm 5{ }^{\circ} \mathrm{C}$. The solidus curve falls from this temperature to about $1340 \pm 5{ }^{\circ} \mathrm{C}$ at 24 mol percent $\mathrm{Na}_{2} \mathrm{O}: 76 \mathrm{~mol}$ percent $\mathrm{Sb}_{2} \mathrm{O}_{4}$. A two phase region exists between the pyrochlore solid solution and $\mathrm{Sb}_{2} \mathrm{O}_{4}$. An unknown phase was found to occur in the system which could be made approximately single phase by calcining the composition $15 \mathrm{~mol}$ percent $\mathrm{Na}_{2} \mathrm{O}: 85$ mol percent $\mathrm{Sb}_{2} \mathrm{O}_{4}$ at $750^{\circ} \mathrm{C}$ and reheating in a sealed $\mathrm{Pt}$ tube to $1000^{\circ} \mathrm{C}$ for $64 \mathrm{~h}$ in the presence of $\mathrm{PtO}_{2}$. This phase has an as yet unindexed x-ray diffraction pattern with the four strongest lines occurring at $d$ values equal to $2.283,2.798,3.453$, $8.23 \mathrm{~A}$.

In the $\mathrm{Sb}_{2} \mathrm{O}_{4}$ rich portion of the system from 10 percent $\mathrm{Na}_{2} \mathrm{O}$ (or $\mathrm{K}_{2} \mathrm{O}$ ):90 percent $\mathrm{Sb}_{2} \mathrm{O}_{4}$ to 100 percent $\mathrm{Sb}_{2} \mathrm{O}_{4}$ experimental interpretation at or near the liquidus is exceedingly difficult since the conventional picture of solid and liquid is not evident. At the composition $15 \mathrm{~mol}$ percent $\mathrm{Na}_{2} \mathrm{O}$ (or $\mathrm{K}_{2} \mathrm{O}$ ) : $85 \mathrm{~mol}$ percent $\mathrm{Sb}_{2} \mathrm{O}_{4}$, quenched liquid plus solid is evident. From this data the solidus can be delineated. However at or near $\mathrm{Sb}_{2} \mathrm{O}_{4}$, the solid appears to transform to vapor with no indication of the liquid phase. The most likely interpretation of the data is shown in the circular insert in figure 1 , indicating that solid $\mathrm{Sb}_{2} \mathrm{O}_{4}+$ solid pyrochlore ${ }_{88}$ melts to solid pyrochlore $_{\mathrm{ss}}$ and liquid. Within experimental error, the sublimation and eutectic points appear to be at the same temperature and the field $\mathrm{Sb}_{2} \mathrm{O}_{4}+\mathrm{Liq}$ (labeled $\mathrm{S}_{1}+\mathrm{L}$ ) is not seen.

\section{1. $\mathrm{NaSbO}_{3}$}

The compound $\mathrm{NaSbO}_{3}$ was first reported by Schrewelius [1] to be hexagonal, $a=5.316$ and $\mathrm{c}=15.95 \mathrm{~A}$ with an ilmenite structure. This compound was found in the present work to melt congruently at about $1555 \pm 5^{\circ} \mathrm{C}$. No other stable polymorphs were encountered.

\subsection{Pyrochlore Solid Solution}

One intermediate phase, a cubic pyrochlore solid solution was characterized in the system. The compositional range varies from approximately $\mathrm{Na}_{2} \mathrm{O}: 3 \mathrm{Sb}_{2} \mathrm{O}_{4}$ to $3 \mathrm{Na}_{2} \mathrm{O}: 5 \mathrm{Sb}_{2} \mathrm{O}_{4}$ with unit cell dimensions varying from 10.289 to 10.286 A respectively. Since the pyrochlore is a tunnel structure and this pyrochlore is the only sodium containing pyrochlore reported that can be formulated by direct synthesis it was worthy of further study as a possible ionic 


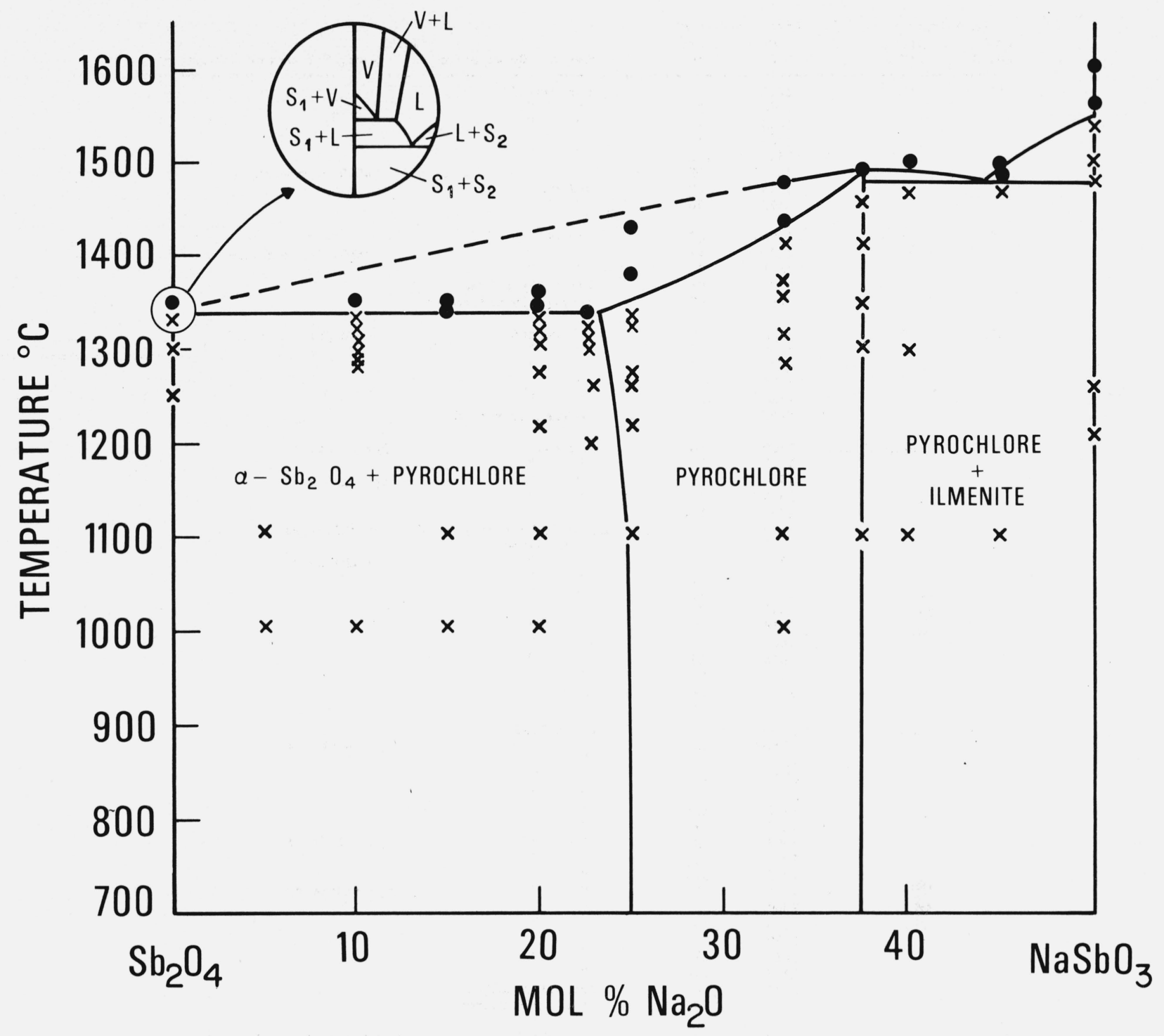

Figure 1. Phase equilibrium diagram for the system $\mathrm{Sb}_{2} \mathrm{O}_{4}-\mathrm{NaSbO}_{3}$.

Not necessarily a true binary system. $\mathrm{L}=$ liquid, $\mathrm{S}=$ solid, $\mathrm{V}=$ vapor, $\mathrm{S}_{1}=\alpha-\mathrm{Sb}_{2} \mathrm{O}_{4}, \mathrm{~S}_{2}=$ pyrochlore.

- - melting.

$x$ - no melting

conductor. For ionic conductivity measurements dense materials were needed and several experiments were conducted with $\mathrm{Na}_{2} \mathrm{O}: 2 \mathrm{Sb}_{2} \mathrm{O}_{4}$ in an effort to determine the stability of the pyrochlore solid solution under high pressure and temperature. Samples in sealed platimun tubes were heated at $1100^{\circ} \mathrm{C}$ and 4000-5000 $\mathrm{psi}^{2}$ for several hours. The resulting specimens are single phase pyrochlore which appear to be very dense. The average density of four measured fragments was $5.26 \pm 0.05 \mathrm{~g} / \mathrm{cm}^{3}$.

2 The use of psi, bar, and kbar follows the current common practice of workers in the field. Note that $1 \mathrm{bar} \equiv 10^{5} \mathrm{~N} / \mathrm{m}^{2}$ (or pascal) $\equiv 10^{6} \mathrm{dyn} / \mathrm{cm}^{2} \equiv 0.9869 \mathrm{~atm} \equiv$ 14.504 psi. The accepted international standard (SI) unit of pressure is the pascal or newton per meter squared.
For ionic conductivity measurements, pellets of $\mathrm{Na}_{2} \mathrm{O}: 2 \mathrm{Sb}_{2} \mathrm{O}_{4}$ (1.9 cm in diameter) were placed in sealed platinum foil envelopes and hot pressed by a commercial company at $1100^{\circ} \mathrm{C}$ and $5,000 \mathrm{psi}$. The pellets were single phase material with a density of 96 percent theoretical (see sec. 6.1). The ionic conductivity of these pellets was measured at NASA Lewis Research Center [8] and they were found to be essentially insulators.

The distribution of the various ions (i.e., $\mathrm{Na}^{+}$, $\left.\mathrm{Sb}^{+3}, \mathrm{Sb}^{+5}, \mathrm{O}^{-2}\right)$ in the $\mathrm{Na}_{2} \mathrm{O}: 2 \mathrm{Sb}_{2} \mathrm{O}_{4}$ specimen is currently being determined at NBS from single crystal structure analysis. Until the results of this analysis are forthcoming it may be assumed that the "lone pair" electrons associated with $\mathrm{Sb}^{+3}$ will not allow the passage of $\mathrm{Na}^{+}$through the channels. 


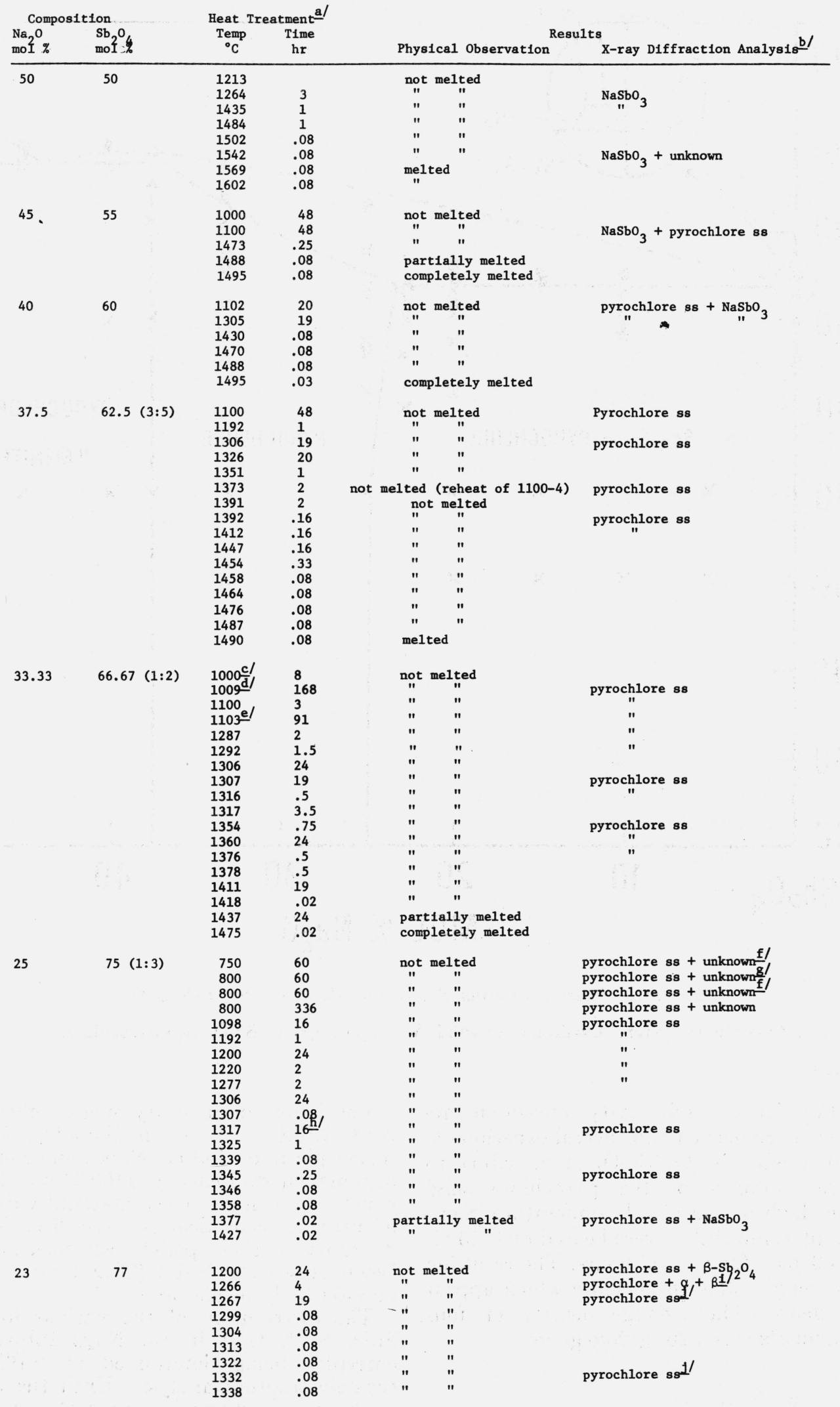

TABLE 1. Experimental data for compositions in the system sodium antimonate-antimony tetroxide 


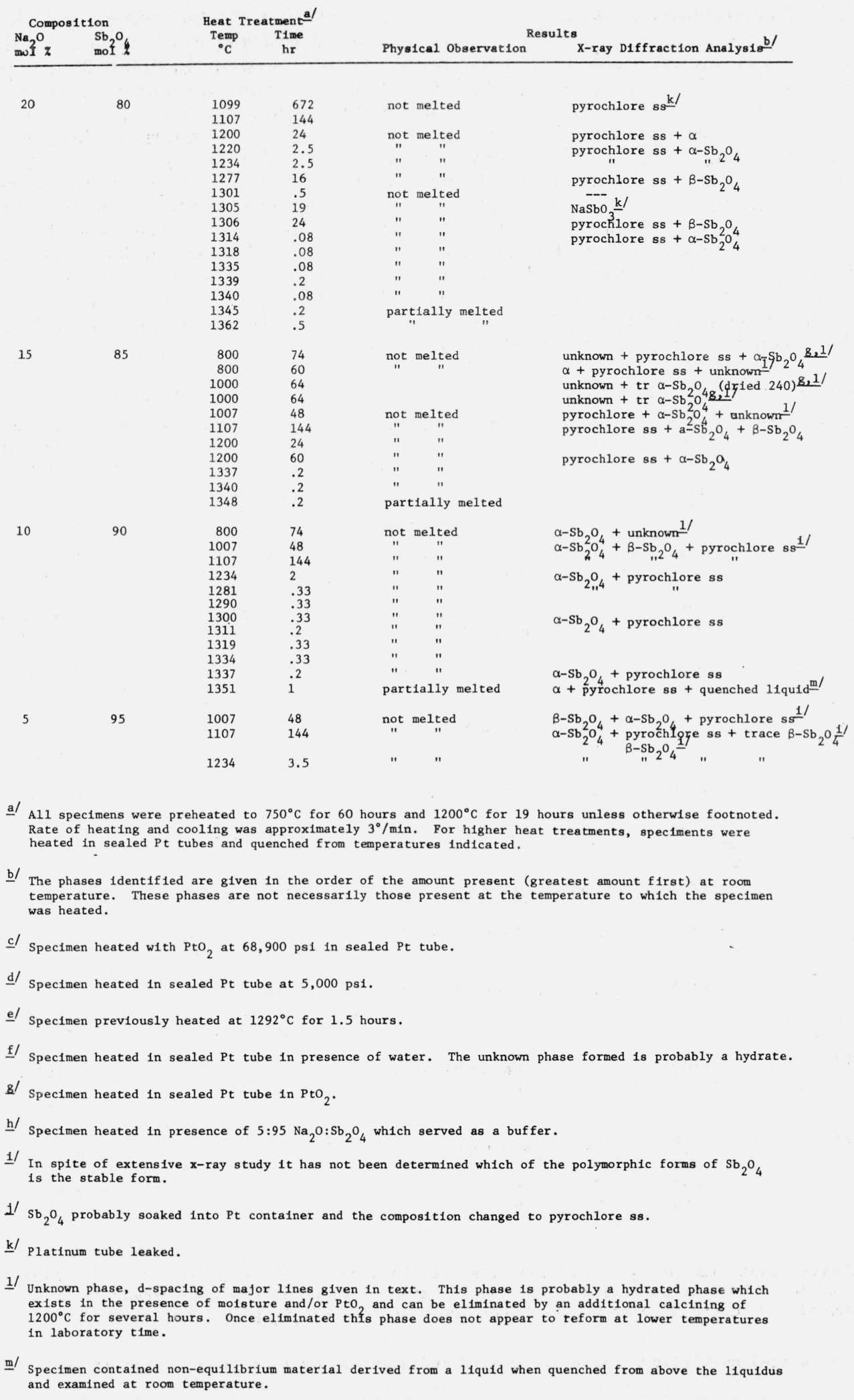

TABLE 1. Experimental data for compositions in the system sodium antimonate-antimony tetroxide-Continued 


\subsection{Polymorphism of $\mathrm{Sb}_{2} \mathrm{O}_{4}$}

Two stable polymorphs of $\mathrm{Sb}_{2} \mathrm{O}_{4}$ have been reported in the literature. They are $\alpha-\mathrm{Sb}_{2} \mathrm{O}_{4}$, which is orthorhombic $[6], a=5.436, b=11.76, c=4.810 \mathrm{~A}$ and $\beta-\mathrm{Sb}_{2} \mathrm{O}_{4}$, which is monoclinic [7], $a=11.905$, $b=4.834, c=5.383 \mathrm{~A}$ and $\beta=101^{\circ} 22^{\prime}$. From table $2 \mathrm{a}$ it can readily be seen that specimens quenched from a temperature-composition region represented on the phase diagram, figure 1 , as $\mathrm{Sb}_{2} \mathrm{O}_{4}+$ pyrochlore may contain either $\alpha-\mathrm{Sb}_{2} \mathrm{O}_{4}$ and/or $\beta-\mathrm{Sb}_{2} \mathrm{O}_{4}$ when quenched from high temperatures and ambient pressures and examined at room temperature. From this seemingly inconsistent data it would appear that $\alpha-\mathrm{Sb}_{2} \mathrm{O}_{4}$ and $\beta-\mathrm{Sb}_{2} \mathrm{O}_{4}$ have a polytypic relationship.
To help resolve this problem a high resolution electron microscope study should be done.

From the data in table $2 \mathrm{~b}$ it appears that the $\beta$ form is the equilibrium high pressure form of $\mathrm{Sb}_{2} \mathrm{O}_{4}$. Insufficient data have been collected to establish if an equilibrium boundary curve exists between $\alpha-\mathrm{Sb}_{2} \mathrm{O}_{4}$ and $\beta-\mathrm{Sb}_{2} \mathrm{O}_{4}$ at various temperatures and pressures. When specimens are sealed and heated under pressure in the presence of $\mathrm{PtO}_{2}$ in either $\mathrm{Pt}$ or $\mathrm{Au}$ tubes single phase $\beta-\mathrm{Sb}_{2} \mathrm{O}_{4}$ is obtained. However when heated under pressure without the $\mathrm{PtO}_{2}$, a two phase specimen results, $\beta-\mathrm{Sb}_{2} \mathrm{O}_{4}$ and the dense high pressure form of $\mathrm{Sb}_{2} \mathrm{O}_{3}$ (valentinite). A similar polytypic relationship probably exists for the two polymorphs of $\mathrm{Sb}_{2} \mathrm{O}_{3}$.

\begin{tabular}{|c|c|c|c|c|c|}
\hline \multirow{2}{*}{$\begin{array}{c}\text { Composition } \\
\text { Starting Material }\end{array}$} & \multicolumn{2}{|c|}{ Heat Treatment } & \multirow[b]{2}{*}{ Environment } & \multicolumn{2}{|l|}{ Results } \\
\hline & $\stackrel{\text { Temp }}{{ }^{\circ} \mathrm{C}}$ & $\begin{array}{c}\text { Time } \\
\mathrm{hr}\end{array}$ & & Physical Observation & $\begin{array}{l}\text { X-ray Diffraction } \\
\text { Analysis }\end{array}$ \\
\hline$\alpha-\mathrm{Sb}_{12}{ }{ }_{4}$ & 1223 & $i^{5}$ & $\begin{array}{l}\text { sealed } \mathrm{Pt} \text { tube } \\
\text { unsealed Pt tube }\end{array}$ & not melted & $\begin{array}{l}\alpha+\operatorname{tr} \beta \\
\alpha\end{array}$ \\
\hline $\mathrm{B}-\mathrm{Sb}_{2} \mathrm{O}_{4}$ & 1223 & $i^{5}$ & $\begin{array}{l}\text { sealed } \mathrm{Pt} \text { tube } \\
\text { unsealed } \mathrm{Pt} \text { tube }\end{array}$ & $\begin{array}{l}\text { not melted } \\
\text { volatilized }\end{array}$ & $\beta+\operatorname{tr} \alpha$ \\
\hline $\begin{array}{l}\beta-\mathrm{Sb}_{2} \mathrm{O}_{4} \\
\alpha-\mathrm{Sb}_{2} \mathrm{O}_{4}\end{array}$ & 1223 & 2 & $\begin{array}{l}\text { sealed } \mathrm{Pt} \text { tube } \\
\text { sealed } \mathrm{Pt} \text { tube }\end{array}$ & not melted & $\begin{array}{l}\beta+\operatorname{tr} \alpha \\
\alpha+\mathrm{Sb}_{2} \mathrm{O}_{3}\end{array}$ \\
\hline $\begin{array}{l}\alpha-\mathrm{Sb}_{2}{ }^{0} \\
\beta-\mathrm{Sb}_{2}{ }^{0} \\
4\end{array}$ & ${ }^{1303}$ & 19 & $\underset{" \text { sealed }}{\mathrm{Pt}}$ tube & not melted & $\underset{\beta}{\beta}+\alpha$ \\
\hline $\begin{array}{l}\alpha-\mathrm{Sb}_{2}{ }^{O} \\
\beta-\mathrm{Sb}_{2}{ }_{2} \mathrm{O}_{4}\end{array}$ & 1327 & $i^{.08}$ & $\underset{" \text { sealed }}{\mathrm{Pt}}$ tube & not melted $^{\prime \prime}$ & $\begin{array}{l}\alpha+\beta \\
\beta+\alpha\end{array}$ \\
\hline$\alpha-\mathrm{Sb}_{2}{ }_{4}$ & 1330 & .25 & sealed $\mathrm{Pt}$ tube & not melted & $\beta+\alpha$ \\
\hline $\begin{array}{l}\alpha-\mathrm{Sb}_{2}{ }^{\mathrm{O}} \\
\beta-\mathrm{Sb}_{2} \mathrm{O}_{4}^{4}\end{array}$ & 1339 & $i^{08}$ & $\underset{" \text { sealed }}{\mathrm{Pt}}$ t tube & not melted & $\begin{array}{l}\alpha+\beta \\
\beta+\alpha\end{array}$ \\
\hline $\mathrm{B}-\mathrm{Sb}_{2} \mathrm{O}_{4}$ & 1345 & .08 & sealed Pt tube & not melted & $\beta+\alpha$ \\
\hline$\alpha-\mathrm{Sb}_{2} \mathrm{O}_{4}$ & 1350 & .08 & sealed Pt tube & $\begin{array}{l}\text { melted (vapor } \\
\text { soaked into } \mathrm{Pt} \text { ) }\end{array}$ & -- \\
\hline $\mathrm{B}-\mathrm{Sb}_{2} \mathrm{O}_{4}$ & 1350 & .08 & sealed $\mathrm{Pt}$ tube & $\begin{array}{l}\text { melted ? } \\
\text { large tabular vapor } \\
\text { grown crystals }\end{array}$ & -- \\
\hline$\alpha-\mathrm{Sb}_{2} \mathrm{O}_{4} \underline{\mathrm{b}} /$ & 1200 & - & $\begin{array}{l}\text { high temperature } \\
\text { x-ray }\end{array}$ & & $\begin{array}{l}\alpha \text { (starting material } \\
\text { remained } \alpha \text { up to } \\
1200^{\circ} \mathrm{C} \text { ) }\end{array}$ \\
\hline $\begin{array}{c}\alpha-\mathrm{Sb}_{2}{ }_{2} \frac{\mathrm{c}}{} \\
" \\
"\end{array}$ & $\begin{array}{l}750 \\
800 \\
900 \\
950\end{array}$ & $\begin{array}{l}24 \\
" 1 \\
" 1\end{array}$ & $\begin{array}{cc}\text { open } & \text { tray } \\
" & " 1 \\
" & "\end{array}$ & & $\begin{array}{l}\alpha \\
" \alpha \\
\alpha+\beta\end{array}$ \\
\hline
\end{tabular}

a/ The phases identified are given in the order of the amount present (greatest amount first) at room temperature. These phases are not necessarily those present at the temperature to which the specimen was heated. $\alpha$ refers to $\alpha-\mathrm{Sb}_{2}{ }_{4}$ polymorph and $\beta$ to the $\beta-\mathrm{Sb}_{2}{ }_{4}$ polymorph.

b/ Material placed on platinum slide and heated and examined by x-ray diffraction at various temperatures.

c/ Poorly crystalline as received $\mathrm{Sb}_{2}{ }^{0} 4$ was heated $750^{\circ} \mathrm{C}-24$ hours and the same specimen which was never ground was reheated at $800^{\circ} \mathrm{C}-24^{2}$ hours, then $900^{\circ} \mathrm{C}-64$ hours and finally $950^{\circ} \mathrm{C}-24$ hours

TABLE 2a. Experimental data for polymorphism in antimony tetroxide 


\section{The System $\mathrm{Sb}_{2} \mathrm{O}_{-}-\mathrm{KSbO}_{3}$}

This system has been examined between the compositional limits of $\mathrm{KSbO}_{3}$ and $\mathrm{Sb}_{2} \mathrm{O}_{4}$. The results are given in the data presented in table 3 from which the phase relationships have been established as shown in figure 2 .

\subsection{Compounds in the System}

The compound $\mathrm{KSbO}_{3}$ with an ilmenite structure $a=5.361, \quad c=18.213$, was previously reported [3] and was found in this work to melt congruently at $1420 \pm 5{ }^{\circ} \mathrm{C}$. A body centered cubic solid solution phase originally reported as $\mathrm{KSbO}_{3}$ [3] has been found to occur metastably at about 47.5 percent $\mathrm{K}_{2} \mathrm{O}$. The $3 \mathrm{~K}_{2} \mathrm{O}: 5 \mathrm{Sb}_{2} \mathrm{O}_{5}$ compound was found to melt congruently at about $1450{ }^{\circ} \mathrm{C}$. The $\mathrm{K}_{2} \mathrm{O}: 2 \mathrm{Sb}_{2} \mathrm{O}_{1}$ compound was found to have a phase transition at about $1000^{\circ} \mathrm{C}$ and to dissociate to pyrochlore plus $3 \mathrm{~K}_{2} \mathrm{O}: 5 \mathrm{Sb}_{2} \mathrm{O}_{5}$ at about $1150{ }^{\circ} \mathrm{C}$. The low temperature form of $\mathrm{K}_{2} \mathrm{O}: 2 \mathrm{Sb}_{2} \mathrm{O}_{5}$, labeled $\mathrm{P}_{1} / c$, represents a monoclinic phase with $a=7.178, \quad b=13.378, \quad c=$ $11.985 \mathrm{~A}$ and $\beta=124^{\circ} 10^{\prime}$. Single crystals of this phase were grown by flux evaporation from the composition $50 \mathrm{~K}_{2} \mathrm{O}: 5 \mathrm{Sb}_{2} \mathrm{O}_{4}: 45 \mathrm{MoO}_{3}$. The unit cell and space group were determined from these crystals and confirmed by least square indexing of the powder diffraction pattern of the low temperature form of the compound $\mathrm{K}_{2} \mathrm{O}: 2 \mathrm{Sb}_{2} \mathrm{O}_{5}$. The pyrochlore solid solution exists at $1150^{\circ} \mathrm{C}$ from about $15 \mathrm{~mol}$ percent $\mathrm{K}_{2} \mathrm{O}: 85$ mol percent $\mathrm{Sb}_{2} \mathrm{O}_{4}$ to greater than $30 \mathrm{~mol}$ percent $\mathrm{K}_{2} \mathrm{O}: 70$ mol percent $\mathrm{Sb}_{2} \mathrm{O}_{4}$. The melting characteristics of these phases have been partially determined as shown in table 3 and figure 2 .

\subsection{Hydroxyl Ion Stabilization of Cubic Potassium Antimonate}

The compound $\mathrm{KSbO}_{3}$ was reported previously as being cubic at ambient conditions after treatment at high temperatures and pressures [9].

In the current work, occasional small amounts of a cubic phase were seen in the x-ray powder diffraction pattern of $\mathrm{KSbO}_{3}$ ilmenite heated at ambient pressure. For these reasons, specimens of $1: 1$ and $3: 5$ mol ratios $\mathrm{K}_{2} \mathrm{O}: \mathrm{Sb}_{2} \mathrm{O}_{4}$ were equilibrated in air at $750{ }^{\circ} \mathrm{C}$ for $60 \mathrm{~h}$ to oxidize and form the phases $\mathrm{KSbO}_{3}$ and $\mathrm{K}_{3} \mathrm{Sb}_{5} \mathrm{O}_{14}$ and then reheated for $1 \mathrm{~h}$ at $1200^{\circ} \mathrm{C}$ to drive off all excess moisture. X-ray diffraction patterns of these specimens showed single phase ilmentite and the $3 \mathrm{~K}_{2} \mathrm{O}: 5 \mathrm{Sb}_{2} \mathrm{O}_{5}$ compound. Portions of these $1200^{\circ} \mathrm{C}$ calcines were then weighed and mixed in acetone in the appropriate ratios to yield compositions of $46,47,47.5,48$ and $49 \mathrm{~mol}$ percent $\mathrm{K}_{2} \mathrm{O}$. Each of these specimens was dried at $240^{\circ} \mathrm{C}$ for $1 \mathrm{~h}$ and heated in open $\mathrm{Pt}$ tubes at $1200^{\circ} \mathrm{C}$ for 1 h. Only the x-ray pattern of the 46 percent specimen showed a small amount of $3 \mathrm{~K}_{2} \mathrm{O}: 5 \mathrm{Sb}_{2} \mathrm{O}_{5}$, the others contained only the cubic phase. A new specimen of

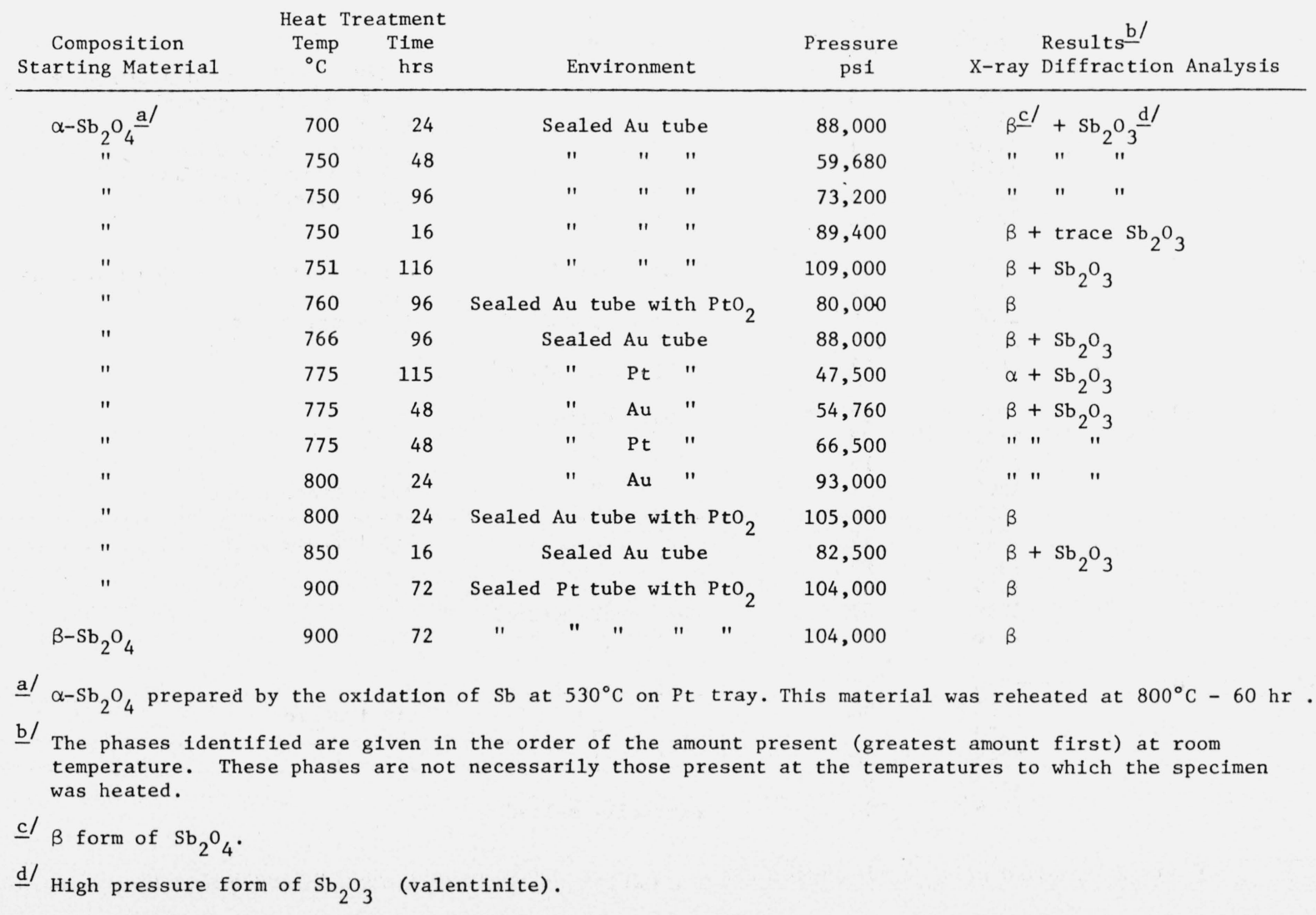

TABLE 2b. Experimental high pressure data for polymorphism in antimony-tetroxide 


\begin{tabular}{|c|c|c|c|c|c|}
\hline \multicolumn{2}{|c|}{ Composition } & \multicolumn{2}{|c|}{ Heat Treatment- $=$} & \multicolumn{2}{|c|}{ Results } \\
\hline $\begin{array}{l}\mathrm{K}_{2} \mathrm{O} \\
\text { Mo1 } \%\end{array}$ & $\begin{array}{l}\mathrm{Sb}_{2} \mathrm{O} \\
\mathrm{MoI} \%\end{array}$ & $\underset{\substack{\text { Temp } \\
{ }^{\circ} \mathrm{C}}}{ }$ & $\begin{array}{l}\text { Time } \\
\mathrm{hr}\end{array}$ & Physical Observation & X-ray Diffraction Analysis \\
\hline 5 & 95 & $\begin{array}{r}950 \\
1168\end{array}$ & $\begin{array}{l}60 \\
48\end{array}$ & not melted & $\begin{array}{l}\text { pyrochlore ss }+\alpha-\mathrm{Sb}_{2} \mathrm{O}_{4}+\beta-\mathrm{Sb}_{2} \mathrm{O}_{4} \text { e/ } \\
\alpha-\mathrm{Sb}_{2} \mathrm{O}_{4}+\beta-\mathrm{Sb}_{2} \mathrm{O}_{4}+\mathrm{pyrochlore}_{\mathrm{ss}} \text { pyror }\end{array}$ \\
\hline 10 & 90 & $\begin{array}{r}950 \\
1168\end{array}$ & $\begin{array}{l}60 \\
48\end{array}$ & not melted & $\underset{"}{\operatorname{pyrochlore}}$ ss $+\underset{"}{\alpha-\mathrm{Sb}_{2} \mathrm{O}_{4}}+\underset{" 1}{\beta-\mathrm{Sb}_{2} \mathrm{O}_{4}}$ \\
\hline 15 & 85 & $\begin{array}{r}853 \\
950 \\
966 \\
1168 \\
1200\end{array}$ & $\begin{array}{r}24 \\
60 \\
4 \\
48 \\
19\end{array}$ & $\begin{array}{cc}\text { not } & \text { melted } \\
" & " \\
" & " \\
" & "\end{array}$ & $\begin{array}{l}\text { pyrochlore ss } \\
\text { pyrochlore ss }+\alpha-\mathrm{Sb}_{2} \mathrm{O}_{4} \\
\text { pyrochlore ss }+\alpha-\mathrm{Sb}_{2}{ }_{4}+\beta-\mathrm{Sb}_{2} \mathrm{O}_{4}\end{array}$ \\
\hline 20 & 80 & $\begin{array}{r}950 \\
1168\end{array}$ & $\begin{array}{l}60 \\
48\end{array}$ & not melted & $\underset{"}{\text { pyrochlore ss }}$ \\
\hline 25 & 75 & $\begin{array}{r}950 \\
1179 \\
1361 \\
1375 \\
1385 \\
1403\end{array}$ & $\begin{array}{r}60 \\
48 \\
.08 \\
.08 \\
.08 \\
.08\end{array}$ & $\begin{array}{l}\text { not melted } \\
" " ~ " \\
\text { partially melted } \\
" ~ \\
\text { completely melted }\end{array}$ & $\begin{array}{l}\mathrm{P} 2 / \mathrm{d}-\mathrm{d} / \\
\text { pyrochlore ss } \\
\text { pyrochlore ss }\end{array}$ \\
\hline 30 & 70 & $\begin{array}{r}950 \\
1178 \\
1366 \\
1380 \\
1382 \\
1399\end{array}$ & $\begin{array}{r}60 \\
48 \\
.08 \\
.08 \\
.08 \\
.08\end{array}$ & $\begin{array}{l}\text { not melted } \\
" " ~ \\
\text { partially melted } \\
\text { " } \\
\text { completely melted }\end{array}$ & $\begin{array}{l}\mathrm{P} 2{ }_{1} / \mathrm{c} / \\
1: 2+\text { pyrochlore ss } \\
\text { pyrochlore ss }+3: 5\end{array}$ \\
\hline 33.33 & 66.67 & $\begin{array}{l}950 \\
950 \\
998 \\
1050 \mathrm{fl} \\
1050 \mathrm{e} \\
1102 \\
1106 \mathrm{f} / \\
1106 \frac{\mathrm{f}}{\mathrm{f} /} \\
1160 \mathrm{f} \\
1179 \mathrm{f} / \\
1214 \mathrm{f} \\
1214\end{array}$ & $\begin{array}{r}60 \\
64 \\
70 \\
168 \\
168 \\
1 \\
64 \\
64 \\
1 \\
48 \\
1 \\
2\end{array}$ & 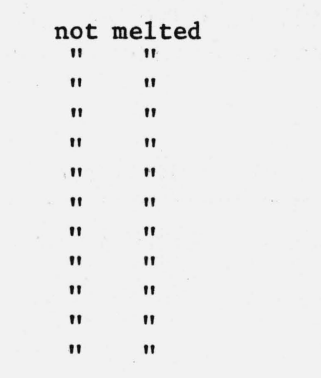 & $\begin{array}{l}3: 5+\mathrm{P} 2{ }_{1} / \mathrm{c} / \\
\mathrm{P} 2{ }_{1} / c^{\mathrm{d}} / \\
1: 2 \frac{\mathrm{c} /}{\mathrm{c} /}+3: 5+\text { pyrochlore } s s+\mathrm{P}{ }_{1} / \mathrm{c} \\
1: 2+3: 5+\text { pyrochlore } \\
1: 2+3: 5 \\
3: 5+\text { pyrochlore ss } \\
1: 2+3: 5+\text { pyrochlore ss } \\
3: 5+\text { pyrochlore ss } \\
" \text { " }\end{array}$ \\
\hline 35 & 65 & $\begin{array}{r}950 \\
1178 \\
1380 \\
1397 \\
1409\end{array}$ & $\begin{array}{l}60 \\
48 \\
.08 \\
.08 \\
.08\end{array}$ & $\begin{array}{l}\text { not melted } \\
\text { " partially melted } \\
" ~ \\
\text { completely melted }\end{array}$ & $\begin{array}{l}\text { pyrochlore }+3: 5+\text { pyrochlore ss } \\
1: 2+3: 5 \\
3: 5+\text { pyrochlore }\end{array}$ \\
\hline 37.5 & 62.5 & $\begin{array}{r}950 \\
1174 \\
1195 \\
1208 \mathrm{e} \\
950- \\
1310 \\
1352 \\
1379 \\
1399 \\
1416\end{array}$ & $\begin{array}{r}60 \\
88 \\
19 \\
1 \\
64 \\
45 \\
.08 \\
.08 \\
.08 \\
.08\end{array}$ & 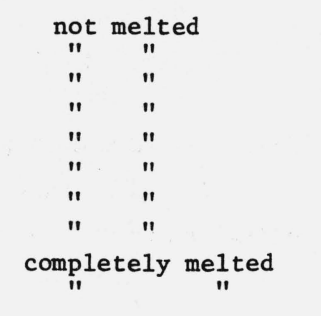 & $\begin{array}{l}3: 5 \\
3: 5+\text { trace cubic } \\
3: 5+\text { trace } 1: 1 \mathrm{~g} /\end{array}$ \\
\hline 40 & 60 & $\begin{array}{l}950 \\
1174 \\
1208 \\
1295 \frac{\mathrm{e}}{\mathrm{e}} \\
1362 \frac{\mathrm{e}}{\mathrm{e} /} \\
1375\end{array}$ & $\begin{array}{l}60 \\
88 \\
1 \\
20 \\
.5 \\
.08\end{array}$ & 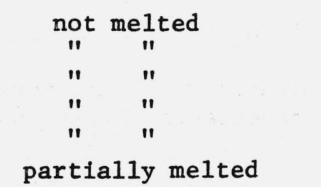 & $\begin{array}{l}3: 5+\underset{" \text { cubic }}{" 1} \\
3: 5+1: 1 \\
" 1 "\end{array}$ \\
\hline
\end{tabular}

TABLE 3. Experimental data for compositions in the system potassium antimonate antimony tetroxide 


\begin{tabular}{|c|c|c|c|c|c|}
\hline \multicolumn{2}{|c|}{ Composition } & \multicolumn{2}{|c|}{ Heat Treatment-a/ } & \multicolumn{2}{|c|}{ Results } \\
\hline $\begin{array}{l}\mathrm{K}_{2} 0 \\
\text { Mo1 \% }\end{array}$ & $\begin{array}{l}\mathrm{Sb}_{2} \mathrm{O} \\
\mathrm{MoI}_{4} \%\end{array}$ & $\begin{array}{l}\text { Temp } \\
{ }^{\circ} \mathrm{C}\end{array}$ & $\begin{array}{c}\text { Time } \\
\text { hr }\end{array}$ & Physical Observation & X-ray Diffraction Analysis \\
\hline 45 & 55 & $\begin{array}{l}950 \\
1174 \\
1208 \mathrm{e} \\
1311 \text { e/ }\end{array}$ & $\begin{array}{r}60 \\
88 \\
1 \\
1\end{array}$ & 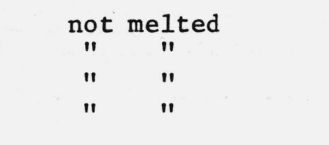 & $\begin{array}{l}1: 1+\text { cubic }+\mathrm{P}_{2} / \mathrm{c} \\
\text { cubic }+3: 5 \\
3: 5+\text { cubic } \\
3: 5+1: 1\end{array}$ \\
\hline 46 & 54 & $1200=\frac{\mathrm{h} /}{}$ & 1 & not melted & cubic $+3: 5$ \\
\hline 47 & 53 & $\begin{array}{l}1194^{\mathrm{h} /} \\
1200\end{array}$ & $\begin{array}{l}3 \\
1\end{array}$ & not melted & $\begin{array}{l}\text { cubic + trace } 3: 5 \\
\text { cubic }\end{array}$ \\
\hline 47.5 & 52.5 & $\begin{array}{l}1212 \frac{\mathrm{h} /}{\mathrm{h} /} \\
1218 \frac{\mathrm{g}}{\mathrm{h}} / \\
1310 \mathrm{~g}\end{array}$ & $\begin{array}{l}88 \\
17 \\
45\end{array}$ & $\begin{array}{l}\text { not melted } \\
" 1 " \\
" ~\end{array}$ & $\begin{array}{l}\text { cubic }+3: 5+1: 1 \\
\text { cubic }+1: 1+3: 5 \\
1: 1\end{array}$ \\
\hline 48 & 52 & $\begin{array}{l}1198 \\
1200 \\
1200 \text { il } \\
1308 \\
1103 \mathrm{c} / \\
1103=\end{array}$ & $\begin{array}{l}3 \\
1 \\
1: 5 \\
.5 \\
1 \\
3\end{array}$ & $\begin{array}{l}\text { not melted } \\
" \text { " } \\
" 1 " \\
" 1 " \\
" \quad "\end{array}$ & $\begin{array}{l}\text { cubic } \\
\text { " } \\
\text { cubic }+3: 5 \text { ilmenite } \\
1: 1 \\
\text { cubic }+ \text { ilmenite }+ \text { pyrochlore } \\
\text { ilmenite }+ \text { pyrochlore }\end{array}$ \\
\hline 49 & 51 & 1200 & 1 & not melted & cubic \\
\hline 50 & 50 & $\begin{array}{r}750 \\
800 \\
921 \\
946 \\
950 \\
1103 \\
1104 \\
1150 \\
1174 \\
1194 \\
1202 \\
1214 \\
1298 \\
1363 \\
1403 \\
1421 \\
1426\end{array}$ & $\begin{array}{r}70 \\
24 \\
1 \\
21 \\
60 \\
1 \\
22 \\
1 \\
88 \\
1 \\
1 \\
1 \\
.5 \\
.5 \\
.08 \\
.08 \\
.08\end{array}$ & 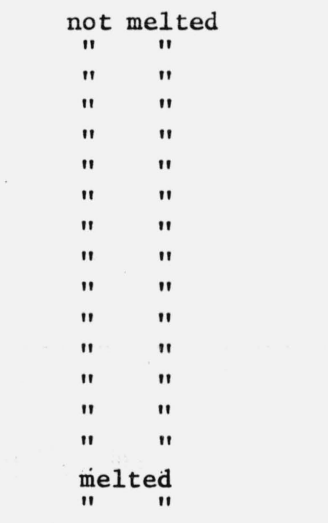 & $\begin{array}{c}\text { ilmenite } \\
\text { " } \\
" \\
" \\
" \\
" \\
" \\
" \\
" \\
"\end{array}$ \\
\hline
\end{tabular}

a/ All specimens were preheated to 500 and $700^{\circ} \mathrm{C}$ for 60 hours unless otherwise footnoted. Rate of heating and cooling were approximately $3^{\circ} / \mathrm{min}$. Specimens were heated in sealed $\mathrm{Pt}$ tubes and quenched from temperatures indicated.

b/ The phases identified are given in order of the amount present (greatest amount first) at room temperature. These phases are not necessarily those present at the temperature to which the specimen was heated. $1: 2-\mathrm{K}_{2} \mathrm{O} \cdot 2 \mathrm{Sb}_{2} \mathrm{O}_{5} ; 3: 5-3 \mathrm{~K}_{2} 0 \cdot 5 \mathrm{Sb}_{2} \mathrm{O}_{5}$ and $1: 1-\mathrm{KSbO}_{3}$ - ilmenite structure.

c/ Non-equilibrium mixture - see Discussion in text.

d/ The phase was indexed from single crystal $x$-ray precession data which has shown the compound is monoclinic space group $\mathrm{P} 2{ }_{1} / c \quad a=7.178, b=13.378, c=11.985, \beta=124^{\circ} 10^{\prime}$.

ef This specimen was previously heated to $500^{\circ}, 700^{\circ}$ and $1200^{\circ} \mathrm{C}-19$ hours in a sealed Pt tube.

f/ Specimen heated in open Pt tube.

g/ Specimen leaked and changed composition.

h/ Composition prepared from a mixture $1: 1$ and $3: 5$ - see text for explanation.

I/ Specimen calcined and examined by x-ray diffraction while in form of pellet.

TABLE 3. Experimental data for compositions in the system potassium antimonate antimony tetroxide-Continued 


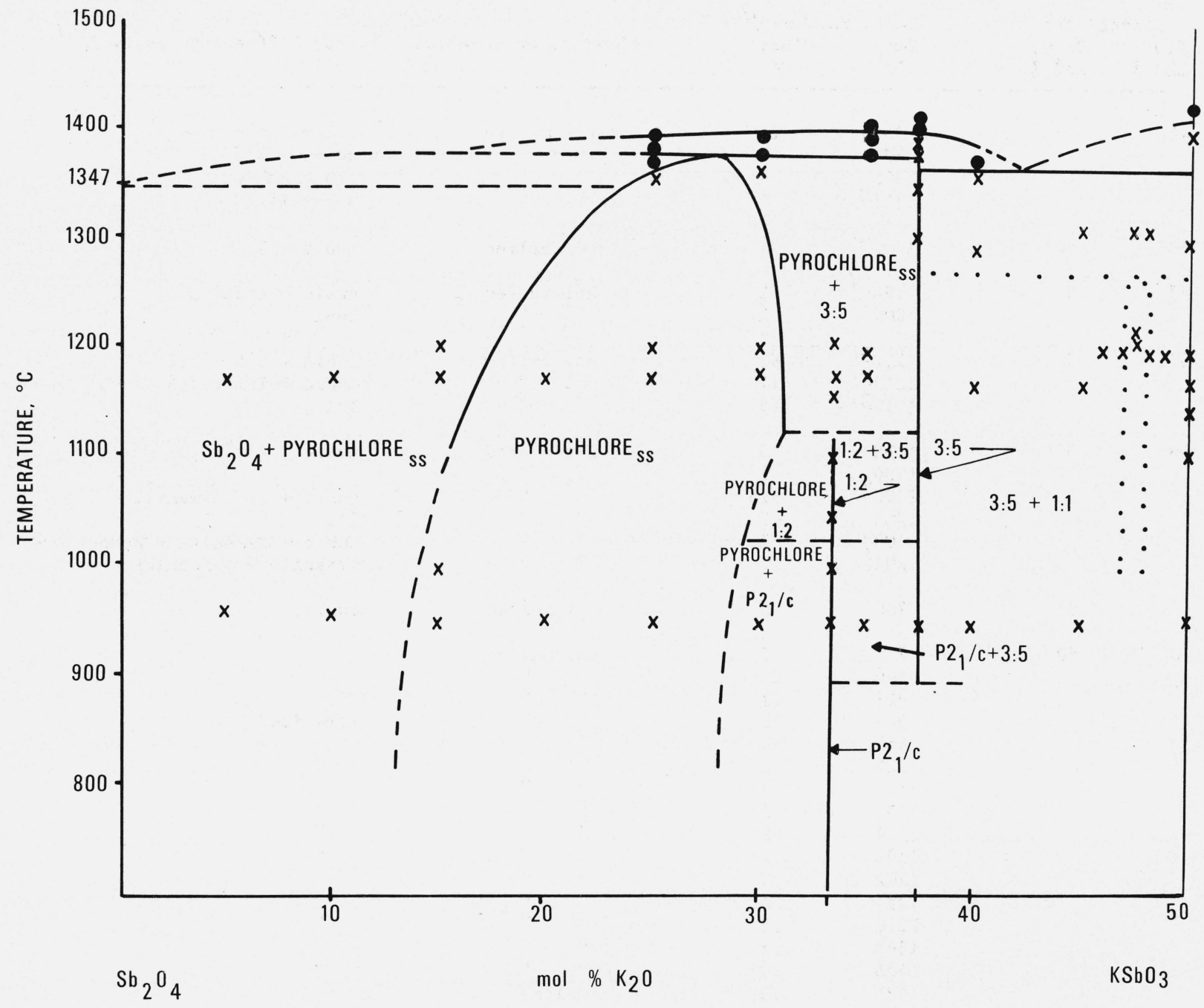

Figure 2. Phase equilibrium diagram for the system $\mathrm{Sb}_{2} \mathrm{O}_{4}-\mathrm{KSbO}_{3}$

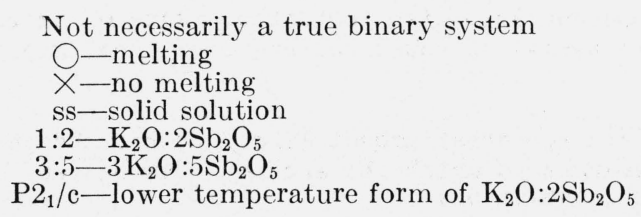

of $48 \mathrm{~mol}$ percent $\mathrm{K}_{2} \mathrm{O}$ was prepared in the same way except the $\mathrm{Pt}$ tube was sealed. After $1 \mathrm{~h}$ at $1200^{\circ} \mathrm{C}$, the x-ray pattern of the specimen showed only about 50 percent cubic. A new specimen of 48 percent $\mathrm{K}_{2} \mathrm{O}$ was prepared by weighing the $1: 1$ and $3: 5$ phases immediately after removal from the $1200^{\circ} \mathrm{C}$ furnace and sealing the material in a flattened $\mathrm{Pt}$ tube within 1-2 min. This tube was then inflated at $1200^{\circ} \mathrm{C}$ for a few minutes and the material mixed by shaking in a "wiggle-birg." The sealed specimen was then heated for $64 \mathrm{~h}$ at $1200{ }^{\circ} \mathrm{C}$. The resultant specimen had exceedingly large grain growth indicating considerable solid state recrystallization but showed no cubic phase. The conclusion is inescapable that access to atmospheric moisture is probably necessary for the formation of the cubic phase at atmospheric pressure.

A paper entitled "Flux Synthesis of Cubic Antimonates" was published by the present authors during the course of this work [10]. In addition to the discovery that the $\mathrm{F}^{-}$ion stabilized the formation of the body centered cubic phase of potassium antimonate it was disclosed that the cubic antimonate could also be obtained by reacting $\mathrm{KSbO}_{3}$ with a small amount of other cations with small radii like $\mathrm{B}^{+3}, \mathrm{Si}^{+4}$, etc. It now appears obvious that in this reaction the boron or silicon (etc.) actually ties up some of the $\mathrm{K}^{+}$ion in a second phase and allows the $\mathrm{K}^{+}$deficient antimonate to react with atmospheric moisture to form the cubic antimonate previously thought to be " $\mathrm{KSbO}_{3}$." 


\section{The Systems of $\mathrm{NaSbO}_{3}$ With Additions}

\subsection{The System $\mathrm{NaSbO}_{3}-\mathrm{NaF}$}

To determine if $\mathrm{NaF}$ additions will stabilize the body-centered cubic phase, similar to the $6 \mathrm{KSbO}_{3}$ : KF-phase [10], NaF was added to $\mathrm{NaSbO}_{3}$ in the ratio of $3 \mathrm{NaSbO}_{3}: \mathrm{NaF}, 4 \mathrm{NaSbO}_{3}: \mathrm{NaF}, \quad 5 \mathrm{NaSbO}_{3}$ : $\mathrm{NaF}$ and $6 \mathrm{NaSbO}_{3}: \mathrm{NaF}$. After heating at $750{ }^{\circ} \mathrm{C}$ and $1000{ }^{\circ} \mathrm{C}$ in sealed $\mathrm{Pt}$ tubes, the x-ray patterns showed only ilmenite and $\mathrm{NaF}$, however after heating at $\sim 1150{ }^{\circ} \mathrm{C}$ all the compositions contained some body centered cubic-type phase. The compositions $3 \mathrm{NaSbO}_{3}: \mathrm{NaF}$ and $4 \mathrm{NaSbO}{ }_{3}: \mathrm{NaF}$, when heated in sealed $\mathrm{Pt}$ tubes at $\sim 1250^{\circ} \mathrm{C}$, did not contain ilmenite and appeared to be the closest to single phase cubic. The small crystals of $4 \mathrm{NaSbO}_{3}: \mathrm{NaF}$ prepared by quenching in a small sealed tube appeared to be well-formed truncated octahedrons. However, the room temperature $\mathrm{x}$-ray diffraction pattern of the material had somewhat diffuse lines, with the exception of the $h 00$ lines which were reasonably sharp, suggesting rhombohedral symmetry. This material was placed on a hot stage microscope slide and analyzed by x-ray diffraction from room temperature up to $220^{\circ} \mathrm{C}$. At $190^{\circ} \mathrm{C}$ the material appeared to start to go cubic and by $220^{\circ} \mathrm{C}$ a good quality cubic x-ray diffraction pattern was obtained $(a=9.353 \mathrm{~A})$. When the material was cooled to room temperature the symmetry was again noncubic. As the $h 00$ lines deteriorate somewhat on cooling, the true symmetry of the room temperature form is probably no higher than monoclinic or triclinic rather than rhombohedral. It was therefore not unreasonable to expect that a body centered cubic phase could be obtained by direct synthesis with $\mathrm{NaF}$ without the necessity for $\mathrm{Na}^{+}$ion exchange.

\subsection{The Ternary System $\mathrm{NaSbO}_{3}: \mathrm{Sb}_{2} \mathrm{O}_{4}: \mathrm{NaF}$}

X-ray diffraction patterns (single crystal and powder) of selected NaF-flux synthesized [11] washed crystals show only a truly cubic body centered phase $(a=9.334 \mathrm{~A})$. It must be postulated that the composition formed by this technique is slightly different from that made essentially single phase at $4 \mathrm{NaSbO}_{3}: \mathrm{NaF}$ in a sealed tube. In an attempt to obtain a fluorine-substituted body centered cubic phase which exists at room temperature the compositions shown in table 4 were prepared and show the reported phases when quenched from $1250^{\circ} \mathrm{C}$. Equilibrium was not obtained in overnight heat treatments at $1200^{\circ} \mathrm{C}$. At $1350^{\circ} \mathrm{C}$ the body centered cubic phase started to decompose. The composition $68 \mathrm{NaSbO}_{3}: 4 \mathrm{Sb}_{2} \mathrm{O}_{4}: 28 \mathrm{NaF}$ (mol \%) was chosen as the best composition for further studies on ceramic procedures [11]. The phases found in the specimens heated at $\sim 1250^{\circ} \mathrm{C}$ are summarized in "equilibrium" diagrams for the quaternary system $\mathrm{NaSbO}_{3}-\mathrm{Sb}_{2} \mathrm{O}_{3}-\mathrm{Sb}_{2} \mathrm{O}_{5}-\mathrm{NaF}$ (fig. 3) and the ternary plane of this system $\mathrm{NaSbO}_{3}-\mathrm{Sb}_{2} \mathrm{O}_{4}-\mathrm{NaF}$ (fig. 4).

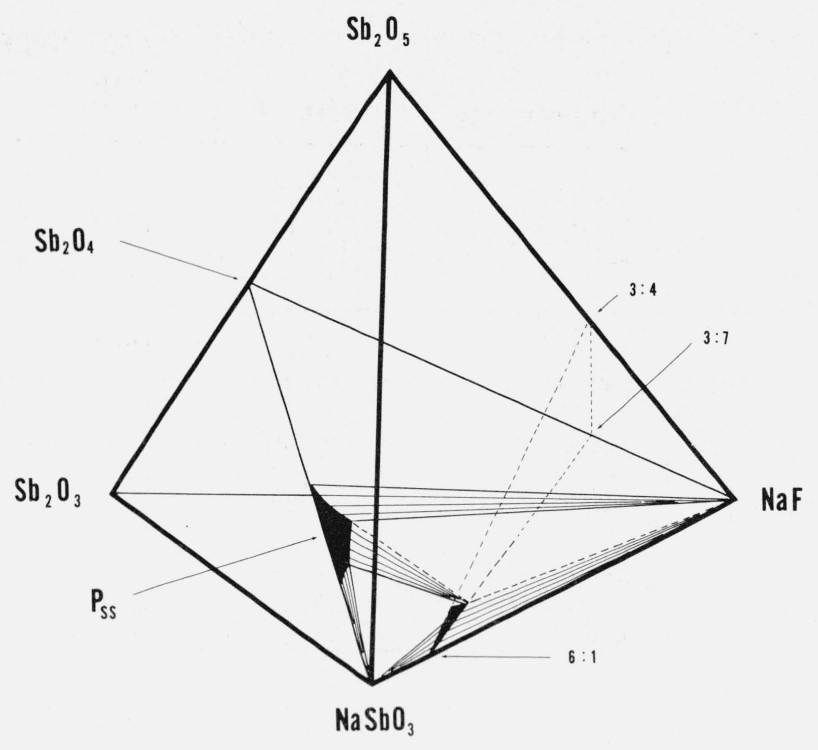

Figure 3. Phase relations in the quaternary system $\mathrm{NaSbO}_{3}-\mathrm{Sb}_{2} \mathrm{O}_{3}-\mathrm{Sb}_{2} \mathrm{O}_{5}-\mathrm{NaF}$.

The join $6: 1-3: 4$ represents the formula

$$
\left[\square_{2+x} \mathrm{Na}_{14-x} \mathrm{~F}_{2}\right]\left[\mathrm{Sb}_{12}{ }^{+5} \mathrm{O}_{36-x} \mathrm{~F}_{x}\right] .
$$

The join $6: 1-3: 7$ represents the formula

$$
\left[\square_{2} \mathrm{Na}_{14} \mathrm{~F}_{2}\right]\left[\mathrm{Sb}_{y}{ }^{+3} \mathrm{Sb}_{12-y}^{+5} \mathrm{O}_{36-(x+2 y)} \mathrm{F}_{(x+2 y)}\right]
$$

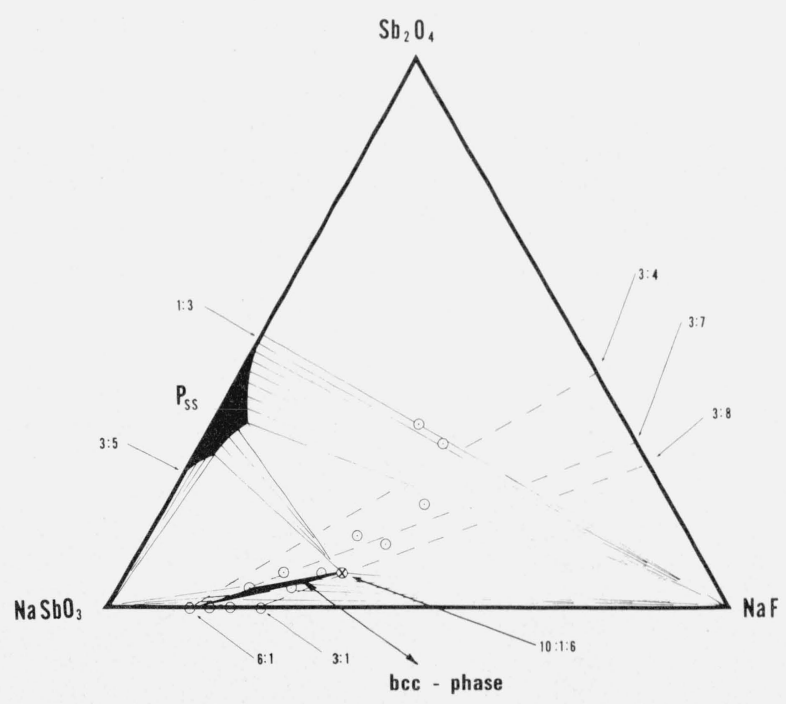

Figure 4. Phase relations in the ternary system $\mathrm{NaSbO}_{3}-\mathrm{Sb}_{2} \mathrm{O}_{4}-\mathrm{NaF}$.

The join $6: 1-3: 4$ represents the formula

$$
\left[\square_{2+x} \mathrm{Na}_{14-x} \mathrm{~F}_{2}\right]\left[\mathrm{Sb}_{12}{ }^{+5} \mathrm{O}_{36-x} \mathrm{~F}_{x}\right]
$$

The join 6:1-3:7 represents the formula

$$
\left[\square_{2} \mathrm{Na}_{14} \mathrm{~F}_{2}\right]\left[\mathrm{Sb}_{y}{ }^{+3} \mathrm{Sb}_{12}{ }^{+5} \mathrm{O}_{36-(x+2 y)} \mathrm{F}_{(x+2 y)}\right]
$$

The join $3: 1-3: 8$ represents the formula

$$
\left[\mathrm{Na}_{16} \mathrm{~F}_{2}\right]\left[\mathrm{Sb}_{2+x}^{+3} \mathrm{Sb}_{10-x}^{+5} \mathrm{O}_{34-2 x} \mathrm{~F}_{2+2 x}\right]+\mathrm{O}_{2} .
$$




\begin{tabular}{|c|c|c|c|c|}
\hline Composition & Mo1 \% & $\begin{array}{l}\text { Heat } \\
\text { Temp } \\
{ }^{\circ} \mathrm{C}\end{array}$ & $\begin{array}{l}\text { atment- } \\
\text { Time } \\
\text { hr }\end{array}$ & $\mathrm{X}$-ray Analysis \\
\hline $\begin{array}{l}\mathrm{NaSbO}_{3} \\
\mathrm{Sb}_{2} \mathrm{O}_{4} \\
\mathrm{NaF}_{4}\end{array}$ & $\begin{array}{r}75.08 \\
3.15 \\
21.77\end{array}$ & 1250 & 19 & single phase distorted cubic \\
\hline $\begin{array}{l}\mathrm{NaSbO}_{3} \\
\mathrm{Sb}_{2} \mathrm{O}_{4} \\
\mathrm{NaF}_{4}\end{array}$ & $\begin{array}{r}67.79 \\
6.25 \\
25.96\end{array}$ & 1250 & 19 & $\begin{array}{l}\text { body centered cubic }+ \\
\text { pyrochlore }+ \text { ilmenite }\end{array}$ \\
\hline $\begin{array}{l}\mathrm{NaSbO}_{3} \\
\mathrm{Sb}_{2} \mathrm{O}_{4} \\
\mathrm{NaF}_{4}\end{array}$ & $\begin{array}{l}53.50 \\
12.34 \\
34.16\end{array}$ & 1250 & 19 & $\begin{array}{l}\text { body centered cubic }+ \\
\text { pyrochlore }+ \text { sodium fluoride }\end{array}$ \\
\hline $\begin{array}{l}\mathrm{NaSbO}_{3} \\
\mathrm{Sb}_{2} \mathrm{O}_{4} \\
\mathrm{NaF}_{4}\end{array}$ & $\begin{array}{l}39.59 \\
18.27 \\
42.14\end{array}$ & 1250 & 19 & $\begin{array}{l}\text { body centered cubic }+ \\
\text { pyrochlore }+ \text { sodium fluoride }\end{array}$ \\
\hline $\begin{array}{l}\mathrm{NaSbO}_{3} \\
\mathrm{Sb}_{2} \mathrm{O}_{4} \\
\mathrm{NaF}_{4}\end{array}$ & $\begin{array}{r}69.05 \\
2.90 \\
28.05\end{array}$ & 1250 & 19 & $\begin{array}{l}\text { body centered cubic }+ \\
\text { trace sodium fluoride }\end{array}$ \\
\hline $\begin{array}{l}\mathrm{NaSbO}_{3} \\
\mathrm{Sb}_{2} \mathrm{O}_{4} \\
\mathrm{NaF}_{4}\end{array}$ & $\begin{array}{l}49.28 \\
11.37 \\
39.35\end{array}$ & 1250 & 19 & $\begin{array}{l}\text { pyrochlore }+ \text { body centered } \\
\text { cubic }+ \text { sodium fluoride }\end{array}$ \\
\hline $\begin{array}{l}\mathrm{NaSbO}_{3} \\
\mathrm{Sb}_{2} \mathrm{O}_{4} \\
\mathrm{NaF}\end{array}$ & $\begin{array}{l}31.20 \\
28.87 \\
39.93\end{array}$ & 1250 & 19 & pyrochlore + sodium fluoride \\
\hline $\begin{array}{l}\mathrm{NaSbO}_{3} \\
\mathrm{Sb}_{2} \mathrm{O}_{4} \\
\mathrm{NaF}_{4}\end{array}$ & $\begin{array}{c}84.62 \\
-- \\
15.38\end{array}$ & 1268 & 19 & ilmenite + cubic \\
\hline $\begin{array}{l}\mathrm{NaSbO}_{3} \\
\mathrm{Sb}_{2} \mathrm{O}_{4} \\
\mathrm{NaF}\end{array}$ & $\begin{array}{r}74.42 \\
2.32 \\
23.26\end{array}$ & $\begin{array}{l}1261 \\
1268\end{array}$ & $\begin{array}{r}1 \\
19\end{array}$ & $\begin{array}{l}\text { distorted cubic }+ \text { ilmenite } \\
\text { distorted cubic }+\mathrm{NaF}\end{array}$ \\
\hline $\begin{array}{l}\mathrm{NaSbO}_{3} \\
\mathrm{Sb}_{2} \mathrm{O}_{4}\end{array}$ & $\begin{array}{r}70.00 \\
3.33 \\
26.67\end{array}$ & 1264 & 1 & cubic + ilmenite \\
\hline $\begin{array}{l}\mathrm{NaSbO}_{3} \\
\mathrm{Sb}_{2} \mathrm{O}_{4} \\
\mathrm{NaF}_{4}\end{array}$ & $\begin{array}{r}65.96 \\
4.26 \\
29.78\end{array}$ & $\begin{array}{l}1266 \\
1267\end{array}$ & $\begin{array}{r}1 \\
19\end{array}$ & $\begin{array}{l}\text { cubic + ilmenite } \\
\text { cubic }+\mathrm{NaF}\end{array}$ \\
\hline $\begin{array}{l}\mathrm{NaSbO}_{3} \\
\mathrm{Sb}_{2} \mathrm{O}_{4} \\
\mathrm{NaF}_{4}\end{array}$ & $\begin{array}{r}62.96 \\
4.94 \\
32.10\end{array}$ & $\begin{array}{l}1266 \\
1267\end{array}$ & $\begin{array}{r}1 \\
19\end{array}$ & $\begin{array}{l}\text { cubic }+\mathrm{NaF} \\
\text { cubic }+\mathrm{NaF}\end{array}$ \\
\hline $\begin{array}{l}\mathrm{NaSbO}_{3} \\
\mathrm{Sb}_{2} \mathrm{O}_{4} \\
\mathrm{NaF}\end{array}$ & $\begin{array}{r}58.82 \\
5.89 \\
35.29\end{array}$ & 1267 & 19 & cubic $+\mathrm{NaF}$ \\
\hline $\begin{array}{l}\mathrm{NaSbO}_{3} \\
\mathrm{Sb}_{2} \mathrm{O}_{4} \\
\mathrm{NaF}\end{array}$ & $\begin{array}{r}68.00 \\
4.00 \\
28.00\end{array}$ & $\begin{array}{l}1000 \\
1252 \\
1265 \\
1265 \\
1265\end{array}$ & $\begin{array}{l}1 \\
16 \\
.1 \\
1.5 \\
72\end{array}$ & $\begin{array}{l}\text { ilmenite }+ \text { trace } \mathrm{NaF} \\
\text { cubic }+ \text { trace ilmenite } \\
\text { cubic }+\mathrm{NaF} \\
\text { cubic }+\mathrm{NaF} \\
\text { cubic }+\mathrm{NaF}\end{array}$ \\
\hline
\end{tabular}

TABLE 4. Experimental data for the ternary system $\mathrm{NaSbO}_{3}-\mathrm{Sb}_{2} \mathrm{O}_{4}-\mathrm{NaF}$ 


\section{Relation of Structural Mechanisms of Non-Stoichiometry to Ionic Conductivity}

It is probably generally accepted that a phase which exhibits unusual ionic conductivity must necessarily be structurally non-stoichiometric. Unfortunately the opposite is not necessarily true. Nevertheless a crystallographic understanding of non-stoichiometric phases is an obvious necessity to the tailoring of new alkali ion conductors. For this reason it is worthwhile to discuss the nature of the non-stoichiometry which has been observed in this study for those phases which seem to be of interest.

\subsection{Pyrochlore Phases}

In the $\mathrm{KTaO}_{3}-\mathrm{WO}_{3}$ system a pyrochlore phase occurs at about the $1: 1$ ratio or $\mathrm{K}_{1.0}[\mathrm{TaW}] \mathrm{O}_{6}[11,12]$. Unfortunately, the pyrochlore in this system transforms to a tetragonal tungsten bronze (TTB) at high termperatures. Although it can be ion exchanged with $\mathrm{Na}^{+}$to produce an ion conducting pyrochlore phase, this phase is not stable above about $450^{\circ} \mathrm{C}$ [11]. The only stable $\mathrm{Na}^{+}$containing pyrochlore is the one in the $\mathrm{Sb}_{2} \mathrm{O}_{4}-\mathrm{NaSbO}_{4}$ system and apparently this one is not a good ionic conductor.

The distribution of $\mathrm{Na}^{+}, \mathrm{Sb}^{+3}, \mathrm{Sb}^{+5}$ and $\mathrm{O}^{-2}$ ions in a pyrochlore single crystal is currently under evaluation by the Crystallography Section at NBS. However, certain assumptions can be made which may enable us to postulate the approximate distribution. The formula for the compositions observed to result in a pyrochlore structure night be postulated to be $\left[\mathrm{NaSb}^{+3}\right] \mathrm{Sb}_{2} \mathrm{O}_{7}$ for the $\mathrm{Na} / \mathrm{Sb}$ ratio of $1: 3$. $\left[\mathrm{Na}_{1.33} \mathrm{Sb}_{0.67}^{+3}\right] \mathrm{Sb}_{2} \mathrm{O}_{6.67}$ for $1: 2$, and $\left[\mathrm{Na}_{1.5} \mathrm{Sb}_{0 .}\right] \mathrm{Sb}_{2} \mathrm{O}_{6.5}$ for $3: 5$. However, these compositions do not illustrate the structural nature of pyrochlore nor account for the observation that the "lone pair" electrons associated with $\mathrm{Sb}^{+3}$ will not allow $\mathrm{O}^{-2}$ ions to completely coordinate the antimony and result in apparent vacancies.

The structural formula of pyrochlore should be written as $\left[\mathrm{A}_{2} \mathrm{X}\right]\left[\mathrm{B}_{2} \mathrm{X}_{6}\right]$ to emphasize the fact that the octahedral network of $\mathrm{B}_{2} \mathrm{X}_{6}$ is required to be complete if the structure is to be stable. The $\mathrm{A}_{2} \mathrm{X}$ ions fill the intersecting channels in this $\mathrm{B}_{2} \mathrm{X}_{6}$ framework. In our material the $\mathrm{B}_{2} \mathrm{X}_{6}$ framework must be represented as $\left[\mathrm{Sb}_{2}{ }^{+5} \mathrm{O}_{6}\right]^{-2}$ and must be stoichiometric. All remaining $\mathrm{Na}^{+}$and $\mathrm{O}^{-2}$ ions, as well as $\mathrm{Sb}^{+3}$, must be in the $\left[\mathrm{A}_{2} \mathrm{X}\right]^{+2}$ portion of the formula. All $\mathrm{Sb}^{+5}$ must be in $\mathrm{B}_{2} \mathrm{X}_{6}$ and only $\mathrm{Sb}^{+3}$ in $\mathrm{A}_{2} \mathrm{X}$. Furthermore the maximum number of the sum of $\mathrm{Na}^{+1}, \mathrm{Sb}^{+3}$, excess $\mathrm{O}^{-2}$ (beyond $\mathrm{O}_{6}^{-2}$ ) and "lone pair" electrons cannot exceed three. One can then write the general formula as $\left[\mathrm{A}_{2} \mathrm{O}\right]^{+2}\left[\mathrm{Sb}_{2} \mathrm{O}_{6}\right]^{-2}$ with $\left[\mathrm{A}_{2} \mathrm{O}\right]^{+2}$ equal to

$$
\left[\mathrm{Na}_{2 / k}^{+1}+\mathrm{Na}_{x}{ }^{+1}+\mathrm{Sb}_{k x}{ }^{+3}+\mathrm{O}_{\vartheta}{ }^{-2}+\mathrm{L} \cdot \mathrm{P} \cdot k x\right] \leq 3
$$

where $k$ equals the ratio $\mathrm{Sb} / \mathrm{Na}$. Using the ionic valences and the sum of the ions equal to three, maximum densities can be calculated and compared with the observed to test the structural hypothesis. The maximum density for the $\mathrm{Na} / \mathrm{Sb}$ ratio of $1: 3$ represented by the formula

$$
\left[\mathrm{Na}_{0.917}^{+1} \mathrm{Sb}_{0.75}^{+3} \mathrm{O}_{0.583}^{-2} \square_{0.75}\right]^{+2}\left[\mathrm{Sb}_{2}{ }^{+5} \mathrm{O}_{6}\right]^{-2}
$$

is calculated to be $5.469 \mathrm{~g} / \mathrm{cm}^{3}$.

For the $\mathrm{Na} / \mathrm{Sb}$ ratio of $3: 5$ with the formula

$$
\left[\mathrm{Na}^{+1}{ }_{1.5} \mathrm{Sb}^{+3}{ }_{0.5} \mathrm{O}^{-2}{ }_{0.5} \square_{0.5}\right]^{+2}\left[\mathrm{Sb}_{2} \mathrm{O}_{6}\right]^{-2}
$$

the density is calculated as $5.406 \mathrm{~g} / \mathrm{cm}^{3}$. For the intermediate composition with the $\mathrm{Na} / \mathrm{Sb}$ ratio of $1: 2$ and a formula of $\left[\mathrm{Na}_{1.294}^{+1} \mathrm{Sb}_{0.588}^{+3} \mathrm{O}_{0.529}^{-2} \square_{0.588}\right]^{+2}\left[\mathrm{Sb}_{2} \mathrm{O}_{6}\right]^{-2}$ the maximum density is found to be $5.481 \mathrm{~g} / \mathrm{cm}^{3}$. The density found for our isostatically hot pressed specimens is 96.0 percent of the maximum theoretical density. It should be remembered however that the true theoretical density of any given $\mathrm{Sb} / \mathrm{Na}$ ratio will decrease with decrease in temperature. Thus the densities obtained on our hot pressed specimens are, in all probability, greater than 96 percent of theoretical in view of the expected increased oxidation of the $\mathrm{Sb}$ at the relatively low temperatures involved.

\subsection{Body Centered Cubic Antimonates}

A successful method of synthesizing cubic potassium antimonate by heating in molten KF was published by the present authors [10]. The major reason for the success in obtaining completely single phase fluorine stabilized cubic potassium antimonate is that the $\mathrm{KSbO}_{3}$ ilmenite form is $\mathrm{H}_{2} \mathrm{O}$ soluble and may be easily separated from the cubic material.

An examination of the structural model of the octahedral framework of the body centered cubic antimonate phase suggests that this structure must always have some anion (X) occupancy in the 000 and $1 / 21 / 2 \quad 1 / 2$ positions. The structural formula thus appears to be $\left[\mathrm{A}_{16} \mathrm{X}_{2}\right]^{+12}\left[\mathrm{Sb}_{12} \mathrm{O}_{36}\right]^{-12}$ with the alkali ion in position (A) located at (or just off) the juncture of the open cages. However, it seems very likely from both structural reasons (bond lengths, etc.) and valency considerations that either or both of the nonframework positions will be nonstoichiometric. Valency considerations require that at least two out of 16 alkali ions must be missing and the structural formula then becomes

$$
\left[\square_{2} \mathrm{~A}_{14} \mathrm{X}_{2}\right]^{+12}\left[\mathrm{Sb}_{12} \mathrm{O}_{36}\right]^{-12}
$$

This formula corresponds to the composition reported by Goodenough, et al. [13] for the single crystal x-ray diffraction analyses of the phase synthesized with KF according to the NBS method [10]:

$$
\mathrm{K}_{12} \mathrm{Sb}_{12} \mathrm{O}_{36} .2 \mathrm{KF} \text { or }\left[\square_{2} \mathrm{~K}_{14} \mathrm{~F}_{2}\right]^{+12}\left[\mathrm{Sb}_{12} \mathrm{O}_{36}\right]^{-12} \text {. }
$$

It seems quite likely, however, that this general formula does not completely account for all of the preparations which have been observed to form this 
structure, whether body centered or primitive. The observation that a primitive phase can be formed, in air, by reaction with atmospheric moisture at a 48:52 ratio suggests that this phase may well have considerably less than 14 alkali ions per unit cell. The formula must be compensated, in this case, by a substitution of a monovalent anion $\left[(\mathrm{OH})^{-}, \mathrm{F}^{-}\right]$ in the octahedral framework. 'The general formula then becomes $\left[\square_{2+x} \mathrm{~A}_{14-x} \mathrm{X}_{2}\right]^{+(12-x)}\left[\mathrm{Sb}_{12} \mathrm{O}_{36-x} \mathrm{X}_{x}\right]^{-(12-x)}$. The composition found at $\sim 48: 52$ in the potassium antimonate system can be written (assuming a ratio of $11: 12 \mathrm{~K} / \mathrm{Sb}$ or $47.826 \% \mathrm{~K}_{2} \mathrm{O}$ ):

or

$$
\mathrm{K}_{22} \mathrm{Sb}_{24} \mathrm{O}_{71}+5 \mathrm{H}_{2} \mathrm{O} \rightarrow \mathrm{K}_{22} \mathrm{Sb}_{24} \mathrm{O}_{66}(\mathrm{OH})_{10}
$$

$$
\left[\square_{5} \mathrm{~K}_{11}(\mathrm{OH})_{2}\right]^{+9}\left[\mathrm{Sb}_{12} \mathrm{O}_{33}(\mathrm{OH})_{3}\right]^{-9}
$$

which also can be described as $6 \mathrm{KSbO}_{3}: 3 \mathrm{Sb}_{2} \mathrm{O}_{5}: 5 \mathrm{KOH}$ The general formula describing the $\mathrm{K}^{+}$containing compositions is then

$$
\left[\square_{2+x} \mathrm{~K}_{14-x} \mathrm{X}_{2}\right]^{+(12-x)}\left[\mathrm{Sb}_{12}{ }^{+5} \mathrm{O}_{36-x} \mathrm{X}^{\prime}\right]^{-(12-x)} .
$$

The above formula contains only pentavalent antimony and apparently does not completely explain the compositions which form a "stable" body centered cubic phase in the system $\mathrm{NaSbO}_{3}$ : $\mathrm{Sb}_{2} \mathrm{O}_{4+x}: \mathrm{NaF}$. The only formula which does not involve the loss or gain of $\mathrm{O}^{-2}$ (or $\mathrm{F}^{-}$) when the $\mathrm{Sb}_{2} \mathrm{O}_{4}$ is added in a sealed tube corresponds to:

$$
\left[\square_{2} \mathrm{Na}_{14} \mathrm{~F}_{2}\right]\left[\mathrm{Sb}_{y}{ }^{+3} \mathrm{Sb}_{12-\nu}^{+5} \mathrm{O}_{36-2 \nu} \mathrm{F}_{2 y}\right]
$$

which is represented by the join $6: 1-3: 7$ on figures 3 and 4 . There is really no place in the framework structure for $\mathrm{Sb}^{+3}$ and it is difficult to believe that octahedrally coordinated antimony can be $\mathrm{Sb}^{+3}$. However, for convenience, the formulas can be written involving $\mathrm{Sb}^{+3}$. The new formula would then have two variables:

$$
\left[\square_{2+x} \mathrm{~A}_{14-x} \mathrm{X}_{2}^{\prime}\right]\left[\mathrm{Sb}_{y}{ }^{+3} \mathrm{Sb}_{12-y}^{+5} \mathrm{O}_{36-(x+2 y)} \mathrm{X}_{(x+2 y)}\right]
$$

represented by the plane in the quaternary system $\mathrm{NaSbO}_{3}: \mathrm{Sb}_{2} \mathrm{O}_{3}: \mathrm{Sb}_{2} \mathrm{O}_{5}: \mathrm{NaF}$ bounded by the $6: 1-3: 4$ and $6: 1-3: 7$ joins of figures 3 and 4 . However the single phase region in this system actually appears to contain more $\mathrm{NaF}$ than described by this general formula. Apparently some $\mathrm{O}_{2}$ is evolved in the sealed $\mathrm{Pt}$ tubes, the amount depending on uncontrolled variables such as the amount of free volume in the tube and on changes from the original composition during treatment. The absolute maximum amount of $\mathrm{NaF}$ which can be accommodated structurally by the body centered cubic phase can be described by the formula

$$
\left[\mathrm{Na}_{16} \mathrm{~F}_{2}\right]^{+14}\left[\mathrm{Sb}_{2-x}^{+3} \mathrm{Sb}_{10-x}^{+5} \mathrm{O}_{34-2 x} \mathrm{~F}_{2+2 x}\right]^{-14}
$$

which represents a line in the system shown by the join $3: 1-3: 8$ in figure 4 and involves the evolution of one molecule of gas $\left(\mathrm{O}_{2}\right)$ per formula unit. The results of our investigations so far suggest that the body centered phase approaches this formula as a limit. The composition of the cubic phase in equilibrium with excess $\mathrm{Sb}_{2} \mathrm{O}_{4}$ and molten $\mathrm{NaF}$ actually appears to touch this line at approximately $10 \mathrm{NaSbO}_{3}: \mathrm{Sb}_{2} \mathrm{O}_{4}: 6 \mathrm{NaF}$ or

$$
\left[\mathrm{Na}_{16} \mathrm{~F}_{2}\right]\left[\mathrm{Sb}_{3}{ }^{+3} \mathrm{Sb}_{9}{ }^{+5} \mathrm{O}_{32} \mathrm{~F}_{4}\right]+\mathrm{O}_{2} \text {. }
$$

The single phase distorted cubic material on the binary join $\mathrm{NaSbO}_{3}: \mathrm{NaF}$ appears to have a composition between $6: 1$ and $5: 1$ or approximately $11 \mathrm{NaSbO}_{3}: 2 \mathrm{NaF}$ or

$$
\left[\mathrm{Na}_{14.18} \mathrm{~F}_{2}\right]\left[\mathrm{Sb}_{12} \mathrm{O}_{35.818} \mathrm{~F}_{0.1818}\right]+0.0909 \mathrm{O}_{2} \text {. }
$$

The compositions in the quaternary system thus probably lie on a join between these two end members.

\section{References}

[1] Schrewelius, N., Diss. Stockholm, pp. 144 (1943).

[2] Stewart, D. J., and Knop, O., Can. J. of Chem. 48, 1323 (1970).

[3] Spiegelberg, P., Ark. f. Kemi Mineral. och Geol. 14 [5] (1940) 1 .

[4] Aurivillius, B., Ark. f. Kemi 25, 505 (1964).

[5] Hong, H. Y-P., Preprint from Lincoln Lab, MIT, sponsored in part by NASA Contract C-43205-C (1974).

[6] Dihlstrom, K., Z. Anorg. Allg. Chem. 239, 57 (1938).

[7] Rogers, D., and Skapski, A. C., Proc. Chem. Soc., 400 (1964).

[8] Singer, J., Fielder, W. L., Kautz, H. E., and Fordyce, J. S., Bull. Am. Ceram. Soc. 54, 454 (1975).

[9] Kafalas, J. A., in Solid State Chemistry, Proceedings 5th Materials Research Symposium, Ed. Roth, R. S., and Schneider, S. J., Nat. Bur. Stand. (U.S.), Spec. Publ. 364, 287-293 (July 1972).

[10] Brower, W. S., Minor, D. B., Parker, H. S., Roth, R. S., and Waring, J. L., Mater. Res. Bull. 9, 1045 (1974).

[11] Roth, R. S., Brower, W. S., Parker, H. S., Minor, D. B., and Waring, J. L., NASA Report No. CR-134869, NBSIR 75-754, April 1975. (Available as N 75-31909 from the National Technical Information Services, Springfield, VA 22161.)

[12] Roth, R. S., Parker, H. S., Brower, W. S., and Minor, D. B., NASA Report No. CR-134599, NBSIR 74-440, April 1974. (Available as N 74-20381 from the National Technical Information Services, Springfield, VA 22161.)

[13] Goodenough, J. B., Hong, H. Y-P., and Kafalas, J. A., Ninth Monthly Report, NASA Contract C-43205-C (May 1974).

(Paper 80A5-916) 